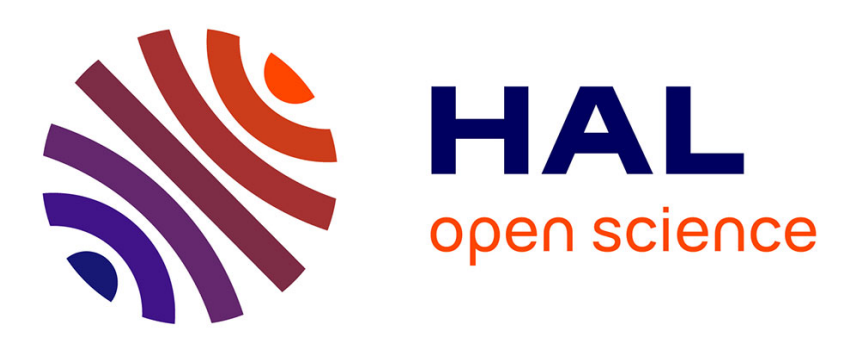

\title{
Local work function measurements of plasma-fluorinated epitaxial graphene
}

\author{
Sonam Sherpa, Jan Kunc, Yike Hu, Galit Levitin, Walt A de Heer, Claire
}

Berger, Dennis Hess

\section{- To cite this version:}

Sonam Sherpa, Jan Kunc, Yike Hu, Galit Levitin, Walt A de Heer, et al.. Local work function measurements of plasma-fluorinated epitaxial graphene. Applied Physics Letters, 2014, 104 (8), pp.081607. 10.1063/1.4866783 . hal-01002911

\section{HAL Id: hal-01002911 \\ https://hal.science/hal-01002911}

Submitted on 7 Jun 2014

HAL is a multi-disciplinary open access archive for the deposit and dissemination of scientific research documents, whether they are published or not. The documents may come from teaching and research institutions in France or abroad, or from public or private research centers.
L'archive ouverte pluridisciplinaire HAL, est destinée au dépôt et à la diffusion de documents scientifiques de niveau recherche, publiés ou non, émanant des établissements d'enseignement et de recherche français ou étrangers, des laboratoires publics ou privés. 


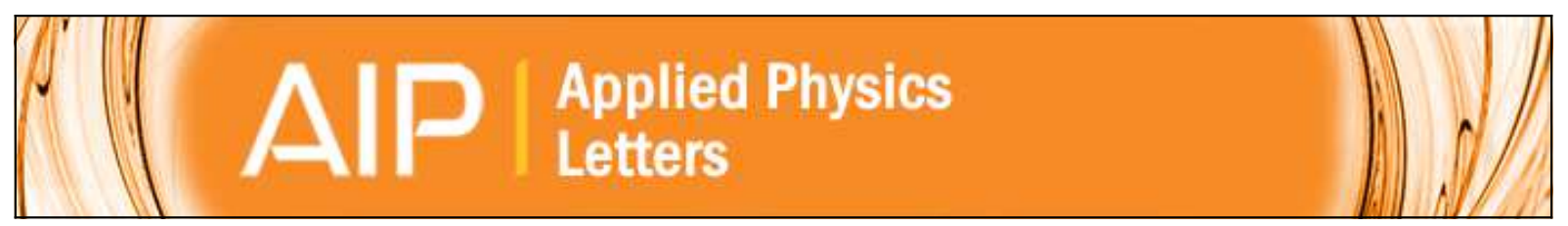

\section{Local work function measurements of plasma-fluorinated epitaxial graphene}

Sonam D. Sherpa, Jan Kunc, Yike Hu, Galit Levitin, Walt A. de Heer, Claire Berger, and Dennis W. Hess

Citation: Applied Physics Letters 104, 081607 (2014); doi: 10.1063/1.4866783

View online: http://dx.doi.org/10.1063/1.4866783

View Table of Contents: http://scitation.aip.org/content/aip/journal/apl/104/8?ver=pdfcov

Published by the AIP Publishing

\section{Articles you may be interested in}

Tuning the work function of graphene by ultraviolet irradiation

Appl. Phys. Lett. 102, 183120 (2013); 10.1063/1.4804289

Effect of the polarity of carbon-fluorine bonds on the work function of plasma-fluorinated epitaxial graphene Appl. Phys. Lett. 101, 111602 (2012); 10.1063/1.4752443

The influence of substrate morphology on thickness uniformity and unintentional doping of epitaxial graphene on $\mathrm{SiC}$

Appl. Phys. Lett. 100, 241607 (2012); 10.1063/1.4729556

Photoelectron spectroscopy studies of plasma-fluorinated epitaxial graphene

J. Vac. Sci. Technol. B 30, 03D102 (2012); 10.1116/1.3688760

Local work function measurements of epitaxial graphene

Appl. Phys. Lett. 93, 133117 (2008); 10.1063/1.2993341

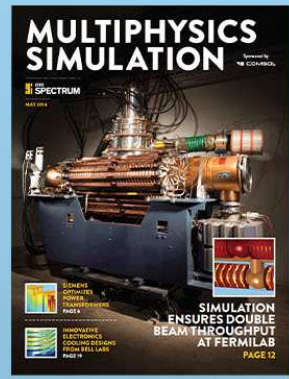

Free online magazine

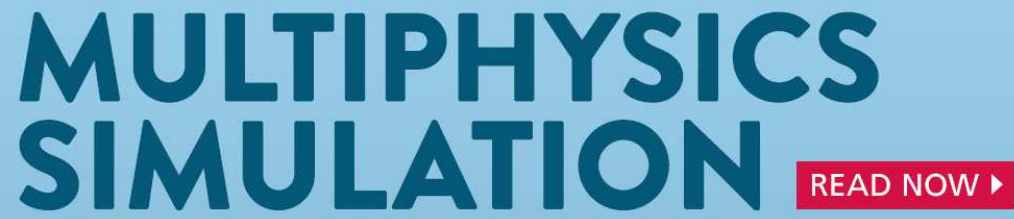




\title{
Local work function measurements of plasma-fluorinated epitaxial graphene
}

\author{
Sonam D. Sherpa, ${ }^{1}$ Jan Kunc, ${ }^{2}$ Yike Hu, ${ }^{2}$ Galit Levitin, ${ }^{1}$ Walt A. de Heer, ${ }^{2}$ Claire Berger, ${ }^{2,3}$ \\ and Dennis W. Hess ${ }^{1, a)}$ \\ ${ }^{1}$ School of Chemical and Biomolecular Engineering, Georgia Institute of Technology, Atlanta, Georgia 30332, \\ USA \\ ${ }^{2}$ School of Physics, Georgia Institute of Technology, Atlanta, Georgia 30332, USA \\ ${ }^{3}$ Institut Néel, CNRS/Univ. J. Fourier, Grenoble, France
}

(Received 26 November 2013; accepted 7 February 2014; published online 24 February 2014)

\begin{abstract}
Plasma-fluorination is an attractive route toward the work function engineering of graphene. The effect of surface topography of epitaxial graphene grown on silicon carbide on the increase in work function after plasma-fluorination was investigated using scanning Kelvin probe microscopy. Results of these studies demonstrate the ability of plasma-treatments to functionalize epitaxial graphene without significant surface roughening. For few-layer epitaxial graphene on the Si-face, work function distribution corresponds to its surface topography. A bimodal distribution is observed before and after fluorination and the separation between the two modes widens after the fluorination. For multi-layer epitaxial graphene on the C-face, no correlation is observed between the work function distribution and the surface topography. After fluorination, the work function is fairly uniform except in few peeled off areas that show a stronger work function contrast. (C) 2014 AIP Publishing LLC. [http://dx.doi.org/10.1063/1.4866783]
\end{abstract}

The ability to control the work function of graphene is critical to its implementation as a transparent electrode material in organic electronic devices. Toward this end, modification of the work function of epitaxial graphene (EG) on $\mathrm{SiC}$ via plasma-fluorination has been demonstrated. ${ }^{1}$ Our studies have also established the correlation between the work function of fluorinated EG and the polarity of carbon-fluorine bonds. ${ }^{2}$ However, factors that determine the polarity of carbon-fluorine bonds in fluorinated EG are not apparent. Previously reported studies on graphite have suggested that a lower concentration of fluorine results in ionic bonding between carbon and fluorine atoms while bonding becomes more covalent as the fluorine concentration increases. ${ }^{3}$ In contrast, carbon-fluorine bonds in the fluorinated EG described in our earlier study display different polarities in spite of similar fluorine concentrations. Understanding the factors that control the polarity of carbon-fluorine bonds in fluorinated EG will provide a technique to tune the electrical properties of graphene by controlling carbon-fluorine bond polarity.

Variation in EG thickness can affect the work function. For instance, the work function difference between singleand bi-layer EG grown on $\mathrm{Si}$-face $6 \mathrm{H}-\mathrm{SiC}$ has been reported be $\sim 135 \mathrm{meV} .{ }^{4}$ This difference in work function results from n-doping from the underlying $\mathrm{SiC}$ substrate and the electronic screening of the subsequent graphene layers. ${ }^{5,6}$ Similarly, effects of surface morphology should also be considered; the effect of topographical features, such as defects, pleats, and pits, on the work function of fluorinated EG has not been studied. In this Letter, we report the results of local work function measurements of EG before and after fluorination by using scanning Kelvin probe microscopy (SKPM). Specifically, roughness of EG measured by atomic force microscopy (AFM) remains unchanged after the fluorination on

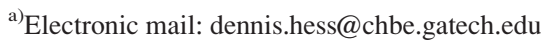

both the $\mathrm{Si}$ - and $\mathrm{C}$-face. For few-layer graphene on the $\mathrm{Si}$ face, the work function distribution matches the surface topography and this correlation is not disrupted by fluorination. For multi-layer epitaxial graphene on the C-face, no correlation is found between the work function distribution and the surface topography before and after fluorination. However, plasma-treatment results in few peeled off graphene areas that display stronger work function contrast.

EG samples were grown on $4 \mathrm{H}-\mathrm{SiC}$ by high-temperature $\left(\sim 1600^{\circ} \mathrm{C}\right)$ annealing in an radio-frequency (RF) furnace by the confinement-controlled sublimation method. ${ }^{7}$ EG samples were fluorinated by $\mathrm{SF}_{6}$ plasma-treatment in an RIE (reactive ion etch) system. This system (Plasma-Therm RIE) operates at a rf of $13.56 \mathrm{MHz}$ and has an electrode diameter of $11 \mathrm{in.}$ All experiments were carried out at room temperature with an rf power of $50 \mathrm{~W}$ and an $\mathrm{SF}_{6}$ partial pressure of 100 mTorr. EG samples were characterized by $\mathrm{x}$-ray photoelectron spectroscopy (XPS) and ultraviolet photoelectron spectroscopy (UPS) to determine elemental composition and estimate the work function, respectively. ${ }^{1}$ XPS and UPS spectra were collected using monochromatized $\mathrm{AlK} \alpha$ radiation $(1486.6 \mathrm{eV})$ and He I radiation $(21.2 \mathrm{eV})$, respectively, in an XPS/UPS system (Axis UltraDLD, Kratos Analytical). The number of graphene layers was determined by measuring the average thickness using ellipsometry (Horiba Jobin Yvon Auto SE). SKPM measures the contact potential difference (CPD) between a conducting AFM tip and the sample, thereby mapping the CPD between the tip and the sample; surface topography data are generated simultaneously via AFM. Since CPD is equal to the difference between the work function of the sample and the tip, the work function of the sample can be mapped with a calibrated tip. However, the absolute calibration of the tip requires well-controlled reference surface and environment and a wide range of values have been reported for PtIr tips (from $4.28 \mathrm{eV}$ (Ref. 8) to $5.5 \mathrm{eV}$ (Ref. 9)). Our own calibration of various surfaces gives a tip work function 

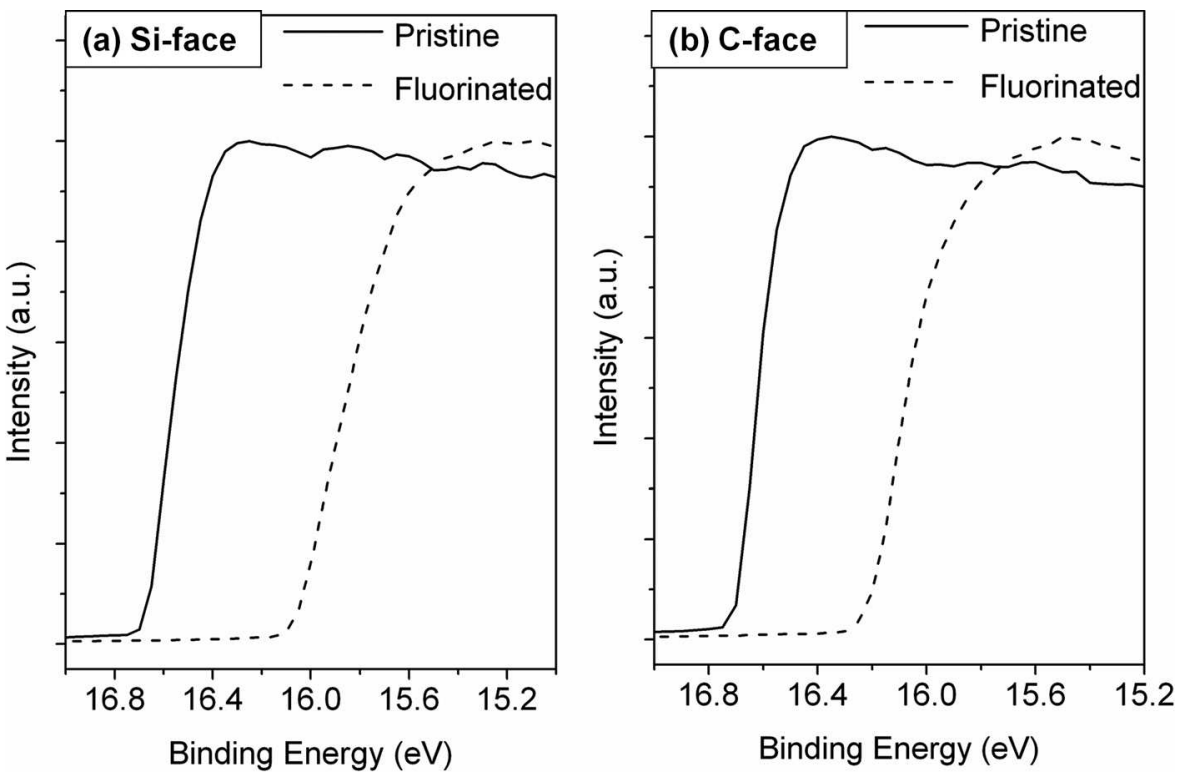

FIG. 1. UPS spectra of pristine and fluorinated EG (a) few-layer EG grown on Si-face $4 \mathrm{H}-\mathrm{SiC}$ and (b) multi-layer ( $\sim 10$ layers) EG grown on $\mathrm{C}$-face $4 \mathrm{H}$ $\mathrm{SiC}$. of $\sim 4.8 \mathrm{eV}$. In view of these results, we opted to measure the average work function of the sample by UPS. Then the histogram of the CPD distribution generated by a SKPM scan was fitted with two Gaussian peaks and the mean of the dominant peak was subtracted from the value of work function measured by UPS to estimate the work function of the tip. We have adopted the same procedure for each sample and work function of the tip ranges between 4.65 and $5 \mathrm{eV}$, which is consistent with the literature and our own calibration. SKPM measurements thus enable the investigation of the effect of surface topography on the work function of fluorinated EG. In this study, SKPM measurements were performed under ambient conditions using a PtIr5 coated $\mathrm{n}^{+}$-silicon tip (XE-70, Park Systems) according to the manufacturer's suggested instrument settings. Specifically, a $1000 \mathrm{mV}$ ac voltage was applied to the tip at the resonance frequency $(\sim 75 \mathrm{kHz})$ with the set-point (amplitude of tip-oscillation in a non-contact mode) equal to $12 \mathrm{~nm}$. This set-point also represents the tip-sample distance. As the tip approaches the sample, the amplitude of oscillation is initially constant. Once the tip enters the net attractive force regime, the oscillation amplitude begins to decrease. Then the tip-sample distance is adjusted to the value of the set-point.
Fig. 1 shows the UPS spectra for few-layer EG on the Si-face before and after fluorination. Work function of EG before fluorination was determined to be $4.45 \mathrm{eV}$ which corresponds to a Fermi level of $\sim 250 \mathrm{meV}$ above the Dirac point (work function of single layer graphene on the Si-face graphene $\sim 4.70 \mathrm{eV}$ (Ref. 10)) and a charge density of $5 \times$ $10^{12} \mathrm{~cm}^{-2}$. This value is consistent with few-layer graphene since the charge density of the top layer decreases with the number of layers due to screening of the interface charge by graphene layers. Fig. 2 compares SKPM images of few-layer EG grown on Si-face before and after fluorination. The surface of few-layer EG before fluorination shown in Fig. 2(a) consists of regions with different height. The rms roughness of $\sim 1.7 \AA$ is estimated by averaging the roughness calculated from five randomly chosen $\sim 1 \mu \mathrm{m}$ AFM line profiles (see supplementary material for the description of how the roughness was calculated ${ }^{16}$ ). As seen in Fig. 2(c), the work function distribution is bimodal and this distribution corresponds to the height distribution (see supplementary material for Fig. 2 that shows the height histogram ${ }^{16}$ ). Two Gaussian peaks were used to fit the histogram of the work function; the separation between the fitted peaks suggests that the work function difference between the two regions is
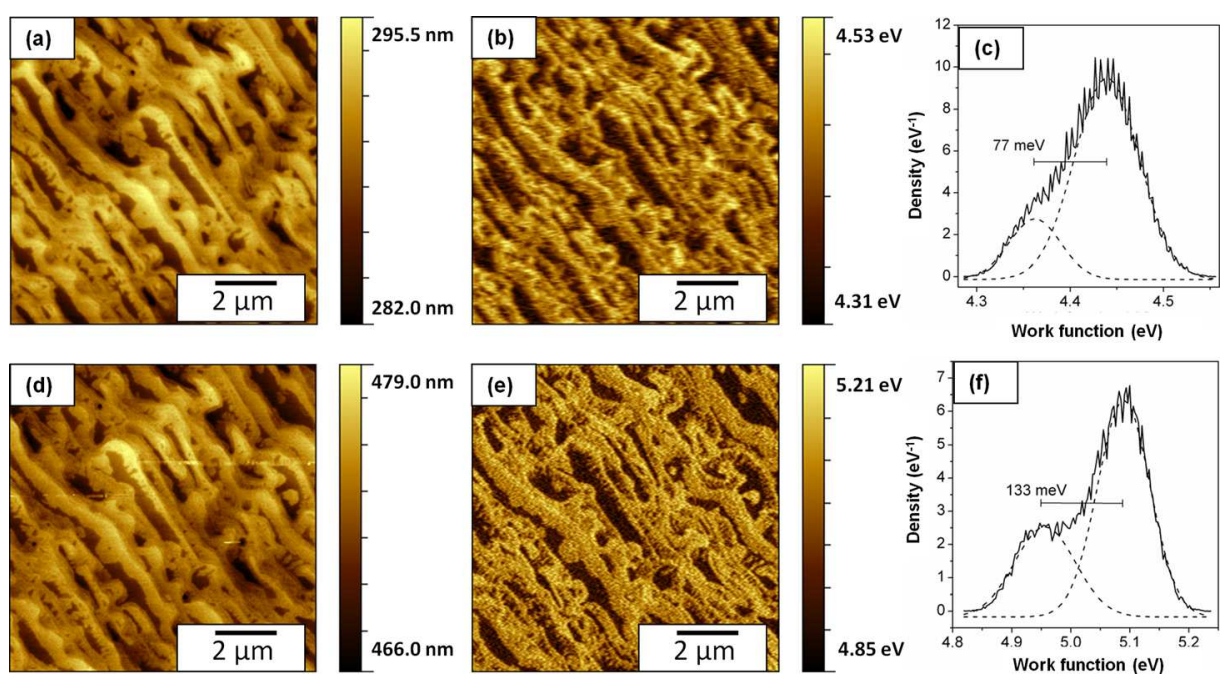

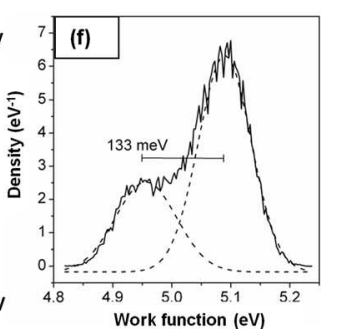

FIG. 2. Surface topography (a), work function map (b), and work function histogram (c) of few-layer EG grown on Si-face $4 \mathrm{H}-\mathrm{SiC}$ before fluorination. Surface topography (d), work function map (e), and work function histogram (f) after fluorination. 

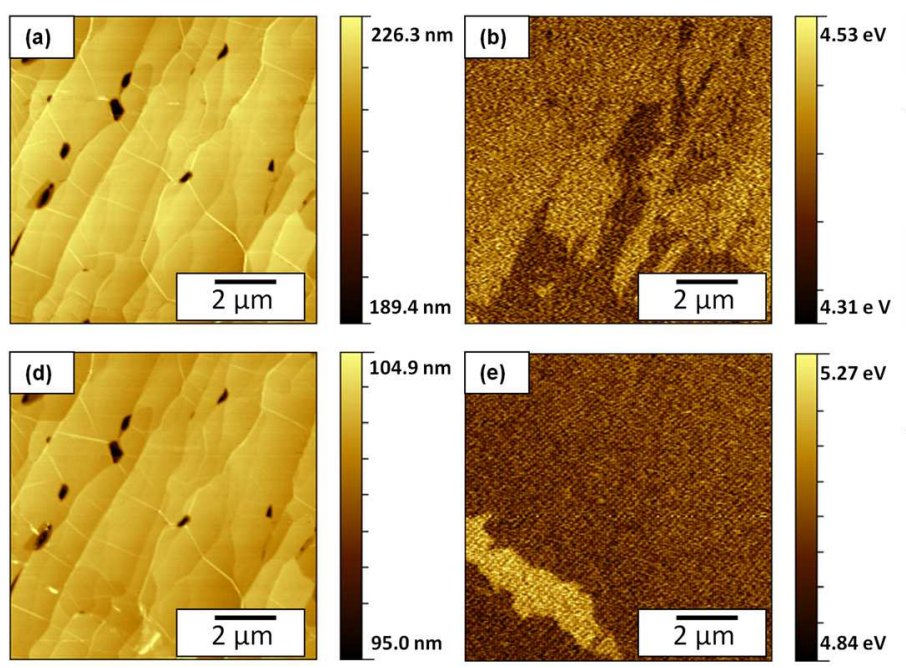

$\sim 77 \mathrm{meV}$. Correlation between the work function and EG thicknesses has been demonstrated previously; ${ }^{4}$ therefore, regions with different height and work function are interpreted as regions with different $\mathrm{EG}$ thicknesses. After $\mathrm{SF}_{6}$ plasma exposure for $30 \mathrm{~s}$, fluorine concentration was $\sim 7 \%$ and the work function increased by $\sim 650 \mathrm{meV}$ [see Fig. $1(\mathrm{a})]$ which is in agreement with our previous report. ${ }^{2}$ Thus, the originally n-doped EG becomes p-doped as expected from the surface dipole induced by carbon-fluorine bond polarity. AFM and SKPM scans were performed over the same region as shown in Figs. 2(d) and 2(e). Fig. 2(d) shows that the topographical features of the EG surface are not noticeably different after the fluorination and the rms roughness of EG is $\sim 2.2 \AA$. These data suggest that the magnitude of the change in the EG roughness after fluorination is on sub-angstrom length scale which can barely be resolved in ambient condition. The work function distribution shown in Fig. 2(e) is similar to the distribution prior to fluorination [Fig. 2(b)], but the difference in work function between the two regions increases to $133 \mathrm{meV}$ [Fig. 2(f)]. Similar SKPM scans were repeated and the average values $(70 \mathrm{meV}$ before fluorination and $140 \mathrm{meV}$ after fluorination) obtained from five different scans validate this increase in separation between the two modes.

Fig. 3 compares SKPM images of multi-layer EG $(\sim 10$ layers) grown on $\mathrm{C}$-face before and after fluorination. Fig. 3(a) shows that the EG surface consists of characteristic topographical features of multi-layer EG including steps, a network of pleats that bound the flat tile-like facets of graphene and a few pits. The rms roughness of EG is $\sim 1.9 \AA$ which may represent the instrument noise in air since the roughness of multi-layer EG has been previously reported to be less than $0.05 \AA$ (Ref. 11) in clean condition. As seen in Fig. 3 (b), there is no correlation between the work function of multi-layer EG and the topographical features. For thick samples, additional EG layers screen the effect of substrateinduced doping. ${ }^{12}$ Hence, we expect that the work function of multi-layer EG also does not depend on EG thickness. Two Gaussian peaks separated by $55 \mathrm{meV}$ were used to fit the histogram of the work function [Fig. 3(c)], which suggests a bimodal work function distribution. However, this work function distribution is not representative of the whole sample. Other regions scanned by SKPM display
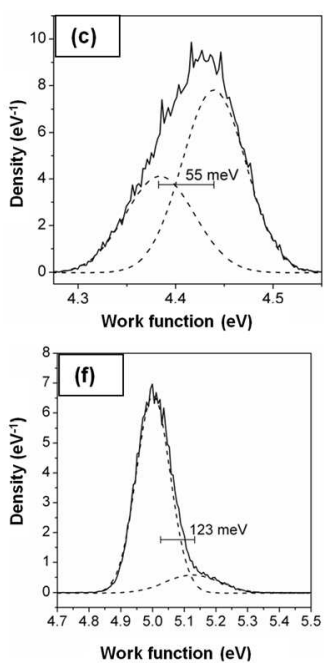

FIG. 3. Surface topography (a), work function map (b), and work function histogram (c) of multi-layer EG $(\sim 10$ layers) grown on $\mathrm{C}$-face $4 \mathrm{H}-\mathrm{SiC}$ before fluorination. Surface topography (d), work function map (e), and work function histogram (f) after fluorination.

homogeneous distribution over a much larger area [Fig. 4]. At present, the reasons for this distribution in work function distribution are not apparent. After $\mathrm{SF}_{6}$ plasma exposure for $30 \mathrm{~s}$, fluorinated EG contains $\sim 8 \%$ fluorine and the increase in work function is $\sim 560 \mathrm{meV}$ [see Fig. 1(b)]. As seen from the comparison between Figs. 3(d) and 3(a), the topographical features remain essentially unchanged after fluorination and the rms roughness of EG is $\sim 1.8 \AA$. However, we also observe few areas as seen in the bottom left corner of Fig. 3(d) where the top layer appears to have been partially exfoliated during the plasma-treatment leaving residues on the surface. The SKPM scan clearly reveals this feature as a bright strip-like area [Fig. 3(e)] of higher work function [ 123 meV from Fig. 3(f)]. During the plasma-treatment, the uppermost EG layer in this region may have been sputtered or partially exfoliated due to non-uniform plasma density that can originate from gradients in the local concentration of fluorine radicals, ${ }^{13}$ fluctuation in ion-fluxes, ${ }^{14}$ or non-uniformities in voltage distribution across the electrode area. ${ }^{15}$ Apart from these seemingly peeled-off areas, the work function distribution after fluorination [Fig. 3(e)] is homogeneous over a much larger area. The localized increase in work function can be explained if a gradient in fluorine concentration exists. Alternatively, a difference in carbon-fluorine bond polarity could also account for these local work function variations. We expect that STM

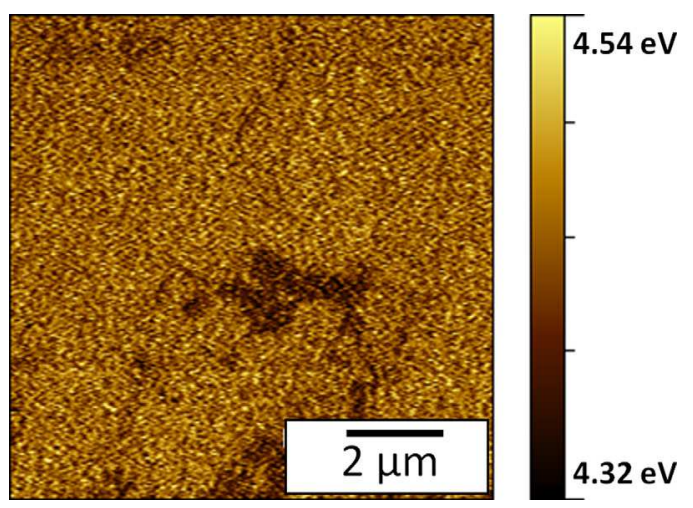

FIG. 4. Work function map of multi-layer EG ( $\sim 10$ layers) grown on C-face $4 \mathrm{H}-\mathrm{SiC}$ before fluorination. 
characterization of fluorinated EG will provide further insight into this localized increase in work function.

In summary, our studies demonstrate that a plasmatreatment can functionalize graphene without significantly roughening the surface which highlights its feasibility as a facile approach for the chemical functionalization of graphene. Work function distribution on few-layer EG on the Si-face corresponds to its surface topography and this bimodal distribution remains intact although the separation between the two modes widens after the fluorination. For multi-layer EG on the C-face, there is no correlation between the work function distribution and its surface topography albeit the emergence of partially exfoliated areas after fluorination results in a localized increase in work function.

We extend our sincere gratitude to Andrei Savu (School of Physics, GA Institute of Technology) for production of epitaxial graphene samples. This work was supported by the W. M. Keck Foundation and the National Science Foundation under MRSEC Grant No. DMR-0820382.

${ }^{1}$ S. D. Sherpa, S. A. Paniagua, G. Levitin, S. R. Marder, M. D. Williams, and D. W. Hess, J. Vac. Sci. Technol. B 30, 03D102 (2012).

${ }^{2}$ S. D. Sherpa, G. Levitin, and D. W. Hess, Appl. Phys. Lett. 101, 111602 (2012).
${ }^{3}$ T. Mallouk, B. L. Hawkins, M. P. Conrad, K. Zilm, G. E. Maciel, and N. Bartlett, Philos. Trans. R. Soc., A 314, 179 (1985).

${ }^{4}$ T. Filleter, K. V. Emtsev, T. Seyller, and R. Bennewitz, Appl. Phys. Lett. 93, 133117 (2008).

${ }^{5}$ C. Berger, Z. Song, T. Li, X. Li, A. Y. Ogbazghi, R. Feng, Z. Dai, A. N. Marchenkov, E. H. Conrad, P. N. First, and W. A. de Heer, J. Phys. Chem. B 108, 19912 (2004).

${ }^{6}$ C. Berger, Z. Song, X. Li, X. Wu, N. Brown, C. Naud, D. Mayou, T. Li, J. Hass, A. N. Marchenkov, E. H. Conrad, P. N. First, and W. A. de Heer, Science 312, 1191 (2006).

${ }^{7}$ W. A. de Heer, C. Berger, M. Ruan, M. Sprinkle, X. Li, Y. Hu, B. Zhang, J. Hankinson, and E. Conrad, Proc. Natl. Acad. Sci. U.S.A. 108, 16900 (2011).

${ }^{8}$ S. Sadewasser, T. Glatzel, M. Rusu, A. Jaüger-Waldau, and M. C. LuxSteiner, Appl. Phys. Lett. 80, 2979 (2002).

${ }^{9} \mathrm{C}$. J. Chen, Introduction to Scanning Tunneling Microscopy (Oxford Science Publications, 2008).

${ }^{10}$ C. Coletti, C. Riedl, D. S. Lee, B. Krauss, L. Patthey, K. von Klitzing, J. H. Smet, and U. Starke, Phys. Rev. B 81, 235401 (2010).

${ }^{11}$ J. Hass, R. Feng, J. Millán-Otoya, X. Li, M. Sprinkle, P. N. First, W. A. de Heer, E. Conrad, and C. Berger, Phys. Rev. B 75, 214109 (2007).

${ }^{12}$ D. Sun, C. Divin, C. Berger, W. A. de Heer, P. N. First, and T. B. Norris, Phys. Rev. Lett. 104, 136802 (2010).

${ }^{13}$ A. G. Nagy, J. Electrochem. Soc. 131, 1871 (1984).

${ }^{14}$ A. Perret, P. Chabert, J.-P. Booth, J. Jolly, J. Guillon, and P. Auvray, Appl. Phys. Lett. 83, 243 (2003).

${ }^{15}$ L. Sansonnens, A. Pletzer, D. Magni, A. A. Howling, C. Hollenstein, and J. P. M. Schmitt, Plasma Sources Sci. Technol. 6, 170 (1997).

${ }^{16}$ See supplementary material at http://dx.doi.org/10.1063/1.4866783 for the description of how the roughness was calculated and Fig. 2 that shows the height histogram. 